\title{
Quality and Safety of Pediatric Inpatient Care in Community Hospitals: A Scoping Review
}

\author{
Jana C Leary, MD, MS ${ }^{1 *}$, Kathleen E Walsh, MD, MS², Rebecca A Morin, MLIS, MAS ${ }^{3}$, \\ Elisabeth G Schainker, MD, MS ${ }^{4}$, JoAnna K Leyenaar, MD, PhD ${ }^{5}$
}

\begin{abstract}
'The Floating Hospital for Children at Tufts Medical Center, Department of Pediatrics, Tufts University School of Medicine, Boston, Massachusetts; ${ }^{2}$ James M Anderson Center for Health Systems Excellence, Department of Pediatrics, Cincinnati Children's Hospital, Cincinnati, Ohio; ${ }^{3}$ Tufts University, Hirsh Health Sciences Library, Boston, Massachusetts; ${ }^{4}$ Franciscan Hospital for Children, Boston, Massachusetts; ${ }^{5}$ The Dartmouth Institute for Health Policy \& Clinical Practice and Department of Pediatrics, Children's Hospital at Dartmouth-Hitchcock Medical Center, Lebanon, New Hampshire.
\end{abstract}

BACKGROUND: Although the majority of children are hospitalized in nonchildren's hospitals, little is known about the quality and safety of pediatric care in community hospitals.

OBJECTIVE: The aim of this study was to conduct a scoping review and synthesize literature on the quality and safety of pediatric inpatient care in United States community hospitals.

METHODS: We performed a systematic literature search in October 2016 to identify pediatric studies that reported on safety, effectiveness, efficiency, timeliness, patientcenteredness, or equity set in general, nonuniversity, or nonchildren's hospitals. We extracted data on study design, patient descriptors, and quality outcomes and assessed the risk of bias using modified Newcastle-Ottawa Scales.

RESULTS: A total of 44 articles met the inclusion criteria. Study designs, patient populations, and quality outcome measures were heterogeneous; only three clinical domains, (1) perinatal regionalization, (2) telemedicine, and (3) imaging radiation, were explored in multiple studies with consistent directionality of results. A total of 30 studies were observational, and 22 studies compared community hospital quality outcomes with other hospital types. The remaining 14 studies reported testing of interventions; 12 showed improved quality of care postintervention. All studies reported an outcome addressing safety, effectiveness, or efficiency, whereas timeliness, patient-centeredness, and equity were infrequently addressed. Risk of bias was moderate or high for $72 \%$ of studies.

CONCLUSIONS: Literature on the inpatient care of children in community hospitals is limited, making it difficult to evaluate healthcare quality. Measures of timeliness, patient-centeredness, and equity are underrepresented. The field would benefit from more multicenter collaborations to facilitate the application of robust study designs and to enable a systematic assessment of individual interventions and community hospital quality outcomes. Journal of Hospital Medicine 2019;14:694-703. () 2019 Society of Hospital Medicine

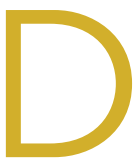

espite efforts to provide high-quality healthcare, Americans die from medical errors each year and many patients do not receive recommended medical care. Risk is particularly acute during times of hospitalization. ${ }^{1-4}$ In response, the Institute of Medicine (IOM, now the Academy of Medicine) has released "Crossing the Quality Chasm: A New Health System for the $21^{\text {st }}$ Century," providing a framework to guide delivery and measurement of high-quality healthcare. ${ }^{5}$

Although the IOM framework has motivated the development of quality improvement (QI) and quality measurement initiatives, relatively few resources have been allocated to im-

*Corresponding Author: Jana C. Leary, MD, MS; E-mail: jleary@tuftsmedicalcenter.org; Telephone: 617-636-4624

Published online first May 10, 2019.

Find Additional Supporting Information in the online version of this article.

Received: April 19, 2019; Revised: June 8, 2019; Accepted: June 13, 2019

() 2019 Society of Hospital Medicine DOI 10.12788/jhm.3268 proving the quality of pediatric inpatient care. ${ }^{6,7}$ The resultant gap in our knowledge of quality and safety of pediatric hospital-based care is further widened by the variability of settings in which children are hospitalized. These settings include freestanding children's hospitals, children's hospitals nested within larger hospitals, and community hospitals, defined as general, nonuniversity, and nonchildren's hospitals. ${ }^{8}$

Although almost three-quarters of children needing hospitalization are cared for outside of freestanding children's hospitals, we know particularly little about the quality and safety of pediatric hospital-based care outside of these settings. ${ }^{6,9}$ Therefore, our scoping review aims to summarize literature regarding the quality and safety of pediatric inpatient care within community hospitals.

\section{METHODS}

We used a scoping review approach because this methodology, by design, is utilized to synthesize evidence and map existing literature and is particularly useful when a body of literature is heterogeneous, rendering a more targeted systematic re- 
TABLE 1. Systematic Approach to Defining a Community Hospital

\begin{tabular}{ll}
\hline Primary Hospital Term Used in Literature & Additional Qualifying Term Required for Inclusion \\
\hline Communitya & None \\
Nontertiary & \\
\hline General & Nonteaching, \\
Nonchildren's & Nonuniversity, \\
Regional medical center & Nonacademic, or \\
Rural & Nontertiary \\
\hline Nonteaching & Nonchildren's, or \\
Nonacademic & Nonspecialty (without specialty designation) \\
Nonuniversity &
\end{tabular}

ancluded unless clearly using the term to describe the American Hospital Association (AHA)/Healthcare Cost and Utilization Project's (HCUP) definition: short-term, non-Federal, general and other hospitals, excluding hospital units of other institutions (eg, prisons). Community hospitals (and HCUP data) include OBGYN, ENT, orthopedic, cancer, pediatric, public, and academic medical hospitals. They exclude hospitals whose main focus is long-term care, psychiatric, and alcoholism and chemical dependency treatment.

view approach infeasible..$^{10}$ This methodology thereby provided an organized approach to answer our broad research question, "What evidence exists regarding the quality and safety of pediatric inpatient care in United States community hospitals?" We followed the scoping review guidelines put forth by the Joanna Briggs Institute and the Preferred Reporting Items for Systematic Reviews and Meta-Analyses (PRISMA) reporting guideline Extension for Scoping Reviews. ${ }^{10,11}$

\section{Data Sources and Search Strategies}

We searched Medline, Medline-In-Process, Embase, the Cochrane Database of Systematic Reviews, the Cumulative Index to Nursing and Allied Health Literature (CINAHL), PsycINFO, and Scopus for studies that reported at least one outcome related to healthcare quality or patient safety and involved pediatric patients (aged $<18$ years) receiving inpatient care at a community hospital. Outcomes included measures from the IOM-defined aims of quality healthcare: (1) safety, (2) effectiveness, (3) efficiency, (4) timeliness, (5) patient-centeredness, and (6) equity (Appendix Table 1). Terms were searched as controlled vocabulary in applicable databases (Medline, Embase, CINAHL, PsycINFO) and as keywords in all databases. Search strategies tailored to each database were developed, tested, and refined in collaboration with a reference librarian. Date parameters for retrieval were set from 1989 to the search date, with the start date chosen to correspond with the establishment of the Agency for Health Care Policy and Research, currently known as the Agency for Healthcare Research and Quality (AHRQ), targeting literature produced in the wake of the $A H R Q$ emphasis on quality in healthcare. Results were limited to articles published in English. Searches were conducted on October 24 and 25, 2016. Complete search strategies can be found in Appendix Methods.

Independent authors performed handsearches of Academic Pediatrics, BMJ Quality \& Safety, Hospital Pediatrics, JAMA Pediatrics, and Pediatrics Quality Reports for the five years preceding the search date (July 2011-October 2016).

\section{Study Selection and Definitions}

To identify studies conducted in community hospitals, we operationalized the definition of community hospital proposed by Percelay. ${ }^{8}$ We used "community", "general", "nonuniversity", and "nonchildren's" as search terms and operationalized these through the addition of qualifying descriptors (Table 1).

Studies were excluded if they (1) were performed outside of the United States, as community hospital definitions may differ by country; (2) included participants aged $>18$ years and did not report any pediatric-specific results; (3) were performed exclusively in tertiary hospitals; (4) evaluated only outpatient or emergency department care; (5) did not report any results specific to community hospitals; (6) did not report any data-driven or parent/patient-reported measures of safety, effectiveness, efficiency, timeliness, patient-centeredness, or equity in their results; and (7) were case reports, case series, editorials, or abstracts without an associated full-text article. We read commentaries and literature reviews related to our objectives and reviewed their references to identify additional articles, but did not include these manuscripts.

Two authors independently reviewed each abstract, and fulltext articles were reviewed if one or more authors determined that the abstract met the inclusion criteria. Two authors then independently reviewed each full-text article to determine whether the article met the criteria for the final review. Disagreements were resolved through consensus after discussion and review with the entire research team, and reasons for exclusion were recorded.

\section{Charting the Results and Data Synthesis}

We used a standardized charting form to collect information regarding study design, community hospital terms, population descriptors, IOM aims of quality healthcare, and outcome measures. To minimize bias in data collection, information from each full-text article was extracted independently by two authors, and differences in extraction were resolved through discussion and re-review among the same two authors. To evaluate the quality of included evidence, two authors ( $\mathrm{JCL}$ and $\mathrm{JKL}$ ) independently assessed the risk of bias for each study using modified Newcastle-Ottawa Quality Assessment Scales (NOS), with disagreements resolved through discussion and re-review among the same two authors. For cohort studies, five of the eight NOS domains were relevant and applied to all included studies (maximum score 6). Using the NOS adapted for cross-sectional studies, six of the seven domains were relevant and applied (maximum score 9). For cross-sectional studies, we defined the risk of bias to be low for scores $\geq 8$, moderate for scores 5-7, and high for scores $\leq 4$, consistent with previous work. ${ }^{12}$ For cohort studies, we similarly defined these strata by scores $\geq 5,3-4$, and $\leq 2$, given the lower maximum score for this study type.

We categorized studies as either observational, defined as cohort or cross-sectional studies of usual healthcare delivery, 
Records identified through database searching:

Medline: 645

CINAHL: 62

Medline in-process: 22

Scopus: 279

Embase: 1,321

PsycINFO: 30

CDSR: 2

Total: 2,361

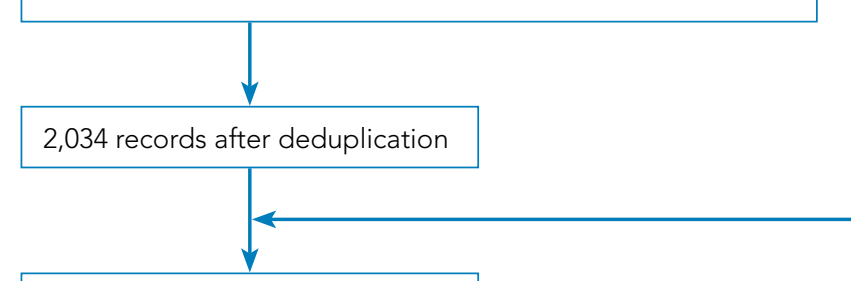

2,068 abstracts identified
Prior lit search, gray lit, expert opinion, hand search

34 articles identified

1,777 abstracts excluded

1,074 Location (non-US)

97 Study design

165 Tertiary

163 Outpatient/ER

237 No obj quality outcome

14 No pediatric results

26 Paper/poster abstract only

1 No community results

248 full text articles excluded

18 Location (non-US)

30 Study design

21 Tertiary

23 Outpatient/ER

51 No obj quality outcome

9 No pediatric results

76 Unclear community terms

20 No community hospital specific results

8 articles identified from search of references

FIG. Flow Diagram of Search Strategy and Article Selection

or interventional, defined as studies evaluating the development and/or implementation of an intervention designed to improve healthcare quality. We further categorized the studies into the following four overarching medical domains: (1) neonatal, (2) pediatric medicine, (3) surgery, or (4) radiology.

\section{RESULTS}

After removal of duplicates, our search identified 2,068 abstracts for screening (Figure). Of these, 1,777 did not meet the inclusion criteria, leaving 291 articles for full-text review. Of these, 43 articles met all the inclusion criteria, and one additional article was included from search of references, resulting in a total of 44 articles.

Study designs, patient populations, and quality outcome measures were heterogeneous. We identified only one randomized controlled trial (RCT). A total of 30 articles were observational studies, 27 of which used retrospective cohort or cross-sectional designs; the remaining three used prospective cohort designs (Table 2). Of these studies, 20 involved multiple 
hospitals, whereas 10 were conducted at a single community hospital. Sample sizes ranged from 29 (single-site) to 107,727 (multisite) patients. Twenty-two studies aimed to compare quality outcomes at community hospitals with other hospital types, of which 16 performed risk-adjusted analyses (detailed findings of observational studies are summarized in Appendix Table 2). The remaining 14 articles were interventional studies (Table 2). Of these, 12 (86\%) reported improvement in quality outcomes after implementation (detailed findings of interventional studies are summarized in Appendix Table 3).

The included studies evaluated quality outcomes addressing all six of the IOM aims of quality healthcare, with safety, effectiveness, and efficiency being the most predominant ( $\mathrm{Ta}$ ble 3). Patient-centeredness and timeliness were infrequently addressed, and only one study assessed equity.

Risk of bias was moderate or high for 27 (69\%) of the observational and interventional cohort studies and four (100\%) of the included cross-sectional studies (Table 2), with the median NOS score being 4 (range: 0-6) for cohort studies (Appendix Table 4) and 4 (range: 3-6) for cross-sectional studies (Appendix Table 5). The higher risk of bias was largely driven by low comparability scores due to inadequate risk adjustment or statistical reporting. Of the 12 studies with low risk of bias, 11 (92\%) were multisite, 9 (75\%) used large regional or national databases, and half reported quality outcomes limited to hospital charges and/or mortality.

\section{Observational Studies \\ Neonatal Medicine}

Five multisite studies focused on neonatal care, ${ }^{13,14,16,19,20}$ of which four examined outcomes associated with the transfer of neonates to or from community hospitals to tertiary care hospitals, such as neonatal morbidity, readmission, completion of preventative health measures and screening, parent satisfaction, and hospital charges. ${ }^{14,16,19,20}$ For example, in a study of extremely premature very low birth weight infants born in Hawaii, Kuo et al. demonstrated that the odds of retinopathy of prematurity was 2.9 times higher for infants born at a community hospital and transported to their tertiary center compared with those inborn at the tertiary center $(P=.02) .{ }^{16}$ The fifth study examined neonatal mortality by site of birth, demonstrating that among infants with birth weights less than 2,000 g, birth at a hospital with a community neonatal intensive care unit (NICU) was associated with 1.4 times higher odds of risk-adjusted mortality compared with birth at a regional NICU $(P<.001) .{ }^{13}$

Three studies evaluated the quality of neonatal care at a single community hospital. ${ }^{15,17,18}$ Quality outcomes were heterogeneous, including utility of rebound bilirubin levels for infants with jaundice, morbidity of neonates requiring mechanical ventilation, and provision of breastfeeding advice to mothers of breastfeeding infants. For instance, Meadow et al. attempted to determine the quality of care for ventilated neonates at one community NICU compared with a tertiary hospital. ${ }^{18}$ They found no difference in days on ventilation or need for home oxygen therapy between the community hospital and the tertiary center, although $P$ values and effect sizes were not report- ed for these outcomes and analyses were not adjusted beyond matching on birthdate and birth weight.

\section{Pediatric Medicine}

Nine multisite studies explored the quality and safety of pediatric medical care across hospital types. ${ }^{21-23,25-30}$ Of these, four were conducted using the Kids' Inpatient Database (KID) ${ }^{28-30}$ or the National Inpatient Sample (NIS), ${ }^{27}$ two were conducted using other national databases, ${ }^{21,25}$ and two were conducted using electronic medical record data. ${ }^{22,26}$ All of the KID and NIS studies examined hospital charges, either alone or in conjunction with mortality. Two of these studies found no differences in risk-adjusted hospital charges between children's hospitals and community hospitals (for burn injuries), ${ }^{28,29}$ whereas two found that hospitalization at community hospitals was associated with lower risk-adjusted charges (for asthma and sepsis). ${ }^{27,30}$ The remaining five studies evaluated diverse quality outcomes such as medication errors, therapeutic drug monitoring, practice guideline compliance, antibiotic prescribing, or hospital-to-home transition summary scores. ${ }^{21-23,25,26}$

Three single-site studies examined quality outcomes for pediatric medical patients, including mortality, outcome ratings for dehydration, and measures of peripherally inserted central catheter (PICC) safety and effectiveness. 24,31,32 For example, Frank et al. evaluated safety of care in one community hospital pediatric intensive care unit (PICU) compared with a tertiary hospital using the Pediatric Risk of Mortality (PRISM) score. ${ }^{24}$ They reported that the observed number of deaths in their community PICU did not differ significantly from the number of deaths predicted in a tertiary center (23 vs 33, respectively, $P>.2$ ).

\section{Surgery}

Three multisite studies examined quality outcomes among children with surgical conditions, including surgical complications, readmission, and hospital charges. ${ }^{34,35,37}$ For example, Kelley-Quon et al. examined outcomes following surgery in infants with hypertrophic pyloric stenosis and found that infants who received their surgery at community hospitals had twice the odds of a surgical complication compared with those at children's hospitals $(P=.027) .{ }^{34}$ They also examined how the risk of appendiceal perforation differed by hospital type, finding that black children who received their surgery at children's hospitals had twice the odds of appendiceal perforation compared with those who received care at community hospitals. ${ }^{35}$

In addition, two studies evaluated quality and safety outcomes for pediatric surgical care in a single community hospital. ${ }^{33,36}$ For example, Beaty et al. prospectively evaluated the incidence of missed injuries in hospitalized pediatric trauma patients, reporting that the incidence of missed injury was 33\% when admission evaluation was performed by a trauma surgeon alone compared to $11 \%$ when performed by a pediatric doctor or a trauma surgeon and a pediatric doctor together $(P<.001){ }^{33}$

\section{Radiology}

Three multisite studies examined quality and safety outcomes associated with radiographic imaging in community hospitals, 
TABLE 2. Characteristics of Included Studies

First Author

(Year)

Abbreviated Title

Community

Study Design Hospital Term(s) Basic Population Description Quality Outcome Measure(s)

Risk of Bias

Observational Studies

Neonatal Medicine

\begin{tabular}{|c|c|c|c|c|c|c|}
\hline $\begin{array}{l}\text { Cifuentes } \\
(2002)^{13}\end{array}$ & $\begin{array}{l}\text { Mortality in Low Birth Weight } \\
\text { Infants- }\end{array}$ & $\begin{array}{l}\text { Retrospective } \\
\text { cohort }\end{array}$ & Community NICU & $\begin{array}{l}\text { Singleton births with infant birth } \\
\text { weight }<2,000 \mathrm{~g}\end{array}$ & Neonatal mortality & Low \\
\hline $\begin{array}{l}\text { Donohue } \\
(2009)^{14}\end{array}$ & $\begin{array}{l}\text { Convalescent Care of Infants in the } \\
\text { Neonatal Intensive Care Unit- }\end{array}$ & Prospective cohort & $\begin{array}{l}\text { Community } \\
\text { hospital }\end{array}$ & $\begin{array}{l}\text { Very low birth weight infants } \\
\text { who received convalescent care } \\
\text { in a tertiary NICU or a community } \\
\text { hospital }\end{array}$ & $\begin{array}{l}\text { Readmission, completion of preventative health } \\
\text { measures and screening examinations, parent } \\
\text { satisfaction, hospital charges }\end{array}$ & High \\
\hline $\begin{array}{l}\text { Izatt } \\
(1997)^{15}\end{array}$ & $\begin{array}{l}\text { Breastfeeding Counseling by Health } \\
\text { Care Providers }\end{array}$ & Prospective cohort & $\begin{array}{l}\text { Community } \\
\text { hospital }\end{array}$ & $\begin{array}{l}\text { Mother-infant dyads attempting to } \\
\text { breastfeed after birth }\end{array}$ & $\begin{array}{l}\% \text { mothers receiving breastfeeding advice during } \\
\text { postpartum hospitalization }\end{array}$ & High \\
\hline $\begin{array}{l}\text { Kuo } \\
(2012)^{16}\end{array}$ & $\begin{array}{l}\text { Outcomes of Inborn and } \\
\text { Transported Extremely Premature- }\end{array}$ & $\begin{array}{l}\text { Retrospective } \\
\text { cohort }\end{array}$ & $\begin{array}{l}\text { Community } \\
\text { hospital }\end{array}$ & $\begin{array}{l}\text { Inborn and transported neonates } \\
\text { hospitalized in an academic center } \\
\text { NICU }\end{array}$ & $\begin{array}{l}\text { Morbidity with IVH, BPD, NEC, severe ROP, and } \\
\text { survival rates }\end{array}$ & Moderate \\
\hline $\begin{array}{l}\text { Maisels } \\
(2002)^{17}\end{array}$ & $\begin{array}{l}\text { Rebound in Serum Bilirubin Level } \\
\text { Following Intensive Phototherapy }\end{array}$ & $\begin{array}{l}\text { Retrospective } \\
\text { cohort }\end{array}$ & $\begin{array}{l}\text { Community } \\
\text { hospital }\end{array}$ & $\begin{array}{l}\text { Neonates aged }>33 \text { weeks } \\
\text { receiving phototherapy in the } \\
\text { newborn nursery }\end{array}$ & $\begin{array}{l}\% \text { infants with rebound bilirubin measured, \% } \\
\text { infants requiring reinitiation of phototherapy }\end{array}$ & Moderate \\
\hline $\begin{array}{l}\text { Meadow } \\
(1996)^{18}\end{array}$ & $\begin{array}{l}\text { Can and Should Level II Nurseries } \\
\text { Care for Newborns- }\end{array}$ & $\begin{array}{l}\text { Retrospective } \\
\text { cohort }\end{array}$ & $\begin{array}{l}\text { Community } \\
\text { hospital }\end{array}$ & $\begin{array}{l}\text { Neonates requiring mechanical } \\
\text { ventilation }\end{array}$ & $\begin{array}{l}\text { Duration of mechanical ventilation, } \% \text { neonates } \\
\text { requiring home oxygen }\end{array}$ & Low \\
\hline $\begin{array}{l}\text { Phibbs } \\
(1992)^{19}\end{array}$ & $\begin{array}{l}\text { Back Transporting Infants from } \\
\text { Neonatal ICUs- }\end{array}$ & $\begin{array}{l}\text { Retrospective } \\
\text { cohort }\end{array}$ & $\begin{array}{l}\text { Nontertiary; } \\
\text { community } \\
\text { hospital }\end{array}$ & $\begin{array}{l}\text { Neonates back-transported from } \\
\text { a tertiary NICU to community } \\
\text { hospitals }\end{array}$ & Hospital charges & Low \\
\hline $\begin{array}{l}\text { Wall } \\
(2004)^{20}\end{array}$ & $\begin{array}{l}\text { Hospital Factors and Nontransfer of } \\
\text { Small Babies- }\end{array}$ & $\begin{array}{l}\text { Retrospective } \\
\text { cohort }\end{array}$ & $\begin{array}{l}\text { Community } \\
\text { hospital; } \\
\text { nontertiary } \\
\text { hospital }\end{array}$ & $\begin{array}{l}\text { Infants weighing } 500-1,249 \mathrm{~g} \text { born } \\
\text { at nontertiary hospitals }\end{array}$ & Nontransfer of infants $<1,250 \mathrm{~g}$ & Low \\
\hline
\end{tabular}

Pediatric Medicine

\begin{tabular}{|c|c|c|c|c|c|c|}
\hline $\begin{array}{l}\text { Alexander } \\
(2009)^{21}\end{array}$ & $\begin{array}{l}\text { Cardiovascular Medication Errors } \\
\text { in Children }\end{array}$ & Cross-sectional & $\begin{array}{l}\text { Community } \\
\text { hospital }\end{array}$ & $\begin{array}{l}\text { Cardiovascular medication error } \\
\text { reports for children }\end{array}$ & Cardiovascular medication errors & High \\
\hline $\begin{array}{l}\text { Balch } \\
(2015)^{22}\end{array}$ & $\begin{array}{l}\text { Pediatric Vancomycin Dosing: Trends } \\
\text { Over Time- }\end{array}$ & $\begin{array}{l}\text { Retrospective } \\
\text { cohort }\end{array}$ & $\begin{array}{l}\text { Community } \\
\text { hospital }\end{array}$ & $\begin{array}{l}\text { Children who received two or more } \\
\text { doses of vancomycin }\end{array}$ & Rates of therapeutic drug monitoring & Moderate \\
\hline $\begin{array}{l}\text { Conway } \\
(2006)^{23}\end{array}$ & $\begin{array}{l}\text { Variations in Management of } \\
\text { Common Inpatient Pediatric } \\
\text { Illnesses- }\end{array}$ & Cross-sectional & $\begin{array}{l}\text { Community } \\
\text { hospital }\end{array}$ & $\begin{array}{l}\text { Pediatric hospitalists and } \\
\text { community pediatricians }\end{array}$ & $\begin{array}{l}\text { Compliance with evidence-based practices for } \\
\text { common inpatient pediatric illnesses }\end{array}$ & Moderate \\
\hline $\begin{array}{l}\text { Frank } \\
(1992)^{24}\end{array}$ & $\begin{array}{l}\text { Quantitative Quality Assurance in a } \\
\text { Community Hospital- }\end{array}$ & $\begin{array}{l}\text { Retrospective } \\
\text { cohort }\end{array}$ & $\begin{array}{l}\text { Community } \\
\text { hospital }\end{array}$ & $\begin{array}{l}\text { Children hospitalized in a } \\
\text { community PICU }\end{array}$ & Mortality & Moderate \\
\hline $\begin{array}{l}\text { Leyenaar } \\
(2014)^{25}\end{array}$ & Variation in Resource Utilization- & $\begin{array}{l}\text { Retrospective } \\
\text { cohort }\end{array}$ & $\begin{array}{l}\text { Community } \\
\text { hospital; general } \\
\text { community } \\
\text { hospital }\end{array}$ & $\begin{array}{l}\text { Children hospitalized with } \\
\text { community acquired pneumonia }\end{array}$ & $\begin{array}{l}\text { Use of diagnostic tests and narrow-spectrum } \\
\text { antibiotics }\end{array}$ & Moderate \\
\hline $\begin{array}{l}\text { Leyenaar } \\
(2016)^{26}\end{array}$ & $\begin{array}{l}\text { Quality Measures to Assess Care } \\
\text { Transitions for Hospitalized Children }\end{array}$ & $\begin{array}{l}\text { Retrospective } \\
\text { cohort }\end{array}$ & $\begin{array}{l}\text { Community } \\
\text { hospital }\end{array}$ & $\begin{array}{l}\text { Children hospitalized and } \\
\text { discharged home }\end{array}$ & Hospital-to-home transition summary score & Moderate \\
\hline $\begin{array}{l}\text { Meurer } \\
(1998)^{27}\end{array}$ & $\begin{array}{l}\text { Charges for Childhood Asthma by } \\
\text { Hospital Characteristics }\end{array}$ & $\begin{array}{l}\text { Retrospective } \\
\text { cohort }\end{array}$ & $\begin{array}{l}\text { Rural nonteaching } \\
\text { hospital }\end{array}$ & Children hospitalized with asthma & Hospital charges & Low \\
\hline $\begin{array}{l}\text { Myers } \\
(2014)^{28}\end{array}$ & $\begin{array}{l}\text { Where are Lengths of Stay Longer } \\
\text { and Total Charges Higher- }\end{array}$ & $\begin{array}{l}\text { Retrospective } \\
\text { cohort }\end{array}$ & $\begin{array}{l}\text { Nonpediatric } \\
\text { nonteaching } \\
\text { hospital }\end{array}$ & $\begin{array}{l}\text { Children hospitalized with burn } \\
\text { injuries }\end{array}$ & Hospital charges & Low \\
\hline $\begin{array}{l}\text { Myers } \\
(2015)^{29}\end{array}$ & $\begin{array}{l}\text { The Effect of Transfers Between } \\
\text { Health Care Facilities- }\end{array}$ & $\begin{array}{l}\text { Retrospective } \\
\text { cohort }\end{array}$ & $\begin{array}{l}\text { Nonpediatric } \\
\text { nonteaching } \\
\text { hospital }\end{array}$ & $\begin{array}{l}\text { Children hospitalized with burn } \\
\text { injuries }\end{array}$ & Hospital charges & Low \\
\hline $\begin{array}{l}\text { Odetola } \\
(2007)^{30}\end{array}$ & $\begin{array}{l}\text { Patient and Hospital Correlates of } \\
\text { Clinical Outcomes- }\end{array}$ & $\begin{array}{l}\text { Retrospective } \\
\text { cohort }\end{array}$ & $\begin{array}{l}\text { Nonchildren's } \\
\text { nonteaching } \\
\text { hospital }\end{array}$ & $\begin{array}{l}\text { Children hospitalized with sepsis } \\
\text { and organ dysfunction }\end{array}$ & In-hospital mortality, hospital charges & Low \\
\hline $\begin{array}{l}\text { Scherb } \\
(2007)^{31}\end{array}$ & $\begin{array}{l}\text { Outcomes Related to Dehydration } \\
\text { in the Pediatric Population }\end{array}$ & $\begin{array}{l}\text { Retrospective } \\
\text { cohort }\end{array}$ & $\begin{array}{l}\text { Community } \\
\text { hospital }\end{array}$ & $\begin{array}{l}\text { Children hospitalized with } \\
\text { dehydration }\end{array}$ & Nursing-sensitive outcome ratings for dehydration & High \\
\hline $\begin{array}{l}\text { Van Winkle } \\
(2008)^{32}\end{array}$ & $\begin{array}{l}\text { Experience Using Peripherally } \\
\text { Inserted Central Venous Catheters- }\end{array}$ & $\begin{array}{l}\text { Retrospective } \\
\text { cohort }\end{array}$ & $\begin{array}{l}\text { Community } \\
\text { hospital }\end{array}$ & $\begin{array}{l}\text { Children with PICCs placed at a } \\
\text { community hospital for parenteral } \\
\text { antibiotics }\end{array}$ & $\begin{array}{l}\text { Rate of successful completion of IV antibiotics at } \\
\text { home, PICC complications, charges compared to } \\
\text { projected inpatient charges }\end{array}$ & High \\
\hline
\end{tabular}


TABLE 2. Characteristics of Included Studies (continued)

\begin{tabular}{|c|c|c|c|c|c|c|}
\hline $\begin{array}{l}\text { First Author } \\
\text { (Year) }\end{array}$ & Abbreviated Title & Study Design & $\begin{array}{l}\text { Community } \\
\text { Hospital Term(s) }\end{array}$ & Basic Population Description & Quality Outcome Measure(s) & Risk of Bias \\
\hline \multicolumn{7}{|l|}{ Surgery } \\
\hline $\begin{array}{l}\text { Beaty } \\
(2003)^{33}\end{array}$ & $\begin{array}{l}\text { Missed Injuries in Pediatric Trauma } \\
\text { Patients }\end{array}$ & Prospective cohort & $\begin{array}{l}\text { Community } \\
\text { hospital }\end{array}$ & $\begin{array}{l}\text { Children hospitalized after } \\
\text { traumatic injury }\end{array}$ & Missed injuries identified after hospital admission & High \\
\hline $\begin{array}{l}\text { Kelley-Quon } \\
(2012)^{34}\end{array}$ & $\begin{array}{l}\text { Hospital Type Predicts Surgical } \\
\text { Complications- }\end{array}$ & $\begin{array}{l}\text { Retrospective } \\
\text { cohort }\end{array}$ & $\begin{array}{l}\text { Community } \\
\text { hospital }\end{array}$ & $\begin{array}{l}\text { Infants with pyloric stenosis who } \\
\text { underwent pyloromyotomy }\end{array}$ & Surgical complications, readmission & Low \\
\hline $\begin{array}{l}\text { Kelley-Quon } \\
(2013)^{35}\end{array}$ & $\begin{array}{l}\text { Hospital Type as a Metric for Racial } \\
\text { Disparities- }\end{array}$ & $\begin{array}{l}\text { Retrospective } \\
\text { cohort }\end{array}$ & $\begin{array}{l}\text { Community } \\
\text { hospital }\end{array}$ & Children with appendicitis & Appendiceal perforation & Low \\
\hline $\begin{array}{l}\text { Pokala } \\
(2007)^{36}\end{array}$ & $\begin{array}{l}\text { Complicated Appendicitis_- Is } \\
\text { the Laparoscopic Approach } \\
\text { Appropriate- }\end{array}$ & $\begin{array}{l}\text { Retrospective } \\
\text { cohort }\end{array}$ & $\begin{array}{l}\text { Community } \\
\text { hospital }\end{array}$ & $\begin{array}{l}\text { Adults and children requiring } \\
\text { appendectomy for complicated } \\
\text { appendicitis }\end{array}$ & $\begin{array}{l}\text { For pediatric patients specifically: postoperative } \\
\text { intra-abdominal abscess, other postoperative } \\
\text { complications }\end{array}$ & Moderate \\
\hline $\begin{array}{l}\text { Smith } \\
(1999)^{37}\end{array}$ & $\begin{array}{l}\text { Does Pediatric Orthopedic } \\
\text { Subspecialization Affect Hospital } \\
\text { Utilization and Charges? }\end{array}$ & $\begin{array}{l}\text { Retrospective } \\
\text { cohort }\end{array}$ & $\begin{array}{l}\text { Community } \\
\text { hospital }\end{array}$ & $\begin{array}{l}\text { Children hospitalized for treatment } \\
\text { of closed femoral shaft fractures or } \\
\text { slipped capital femoral epiphyses }\end{array}$ & Hospital charges & Moderate \\
\hline \multicolumn{7}{|l|}{ Radiology } \\
\hline $\begin{array}{l}\text { Calvert } \\
(2012)^{38}\end{array}$ & $\begin{array}{l}\text { Variation in computed tomography } \\
\text { radiation dose- }\end{array}$ & Cross-sectional & $\begin{array}{l}\text { Community } \\
\text { hospital }\end{array}$ & $\begin{array}{l}\text { Children with traumatic injury } \\
\text { evaluated with abdominal or pelvic } \\
\mathrm{CT} \text { at a community hospital }\end{array}$ & Radiation dose administered & High \\
\hline $\begin{array}{l}\text { Marin } \\
(2015)^{39}\end{array}$ & $\begin{array}{l}\text { Variation in Pediatric Cervical Spine } \\
\text { Computed Tomography Radiation } \\
\text { Dose Index }\end{array}$ & Cross-sectional & $\begin{array}{l}\text { Community } \\
\text { hospital }\end{array}$ & C-spine CTs performed on children & Volume $\mathrm{CT}$ dose index & High \\
\hline $\begin{array}{l}\text { Reich } \\
(2000)^{40}\end{array}$ & $\begin{array}{l}\text { Use of CT Scan in the Diagnosis of } \\
\text { Pediatric Acute Appendicitis }\end{array}$ & $\begin{array}{l}\text { Retrospective } \\
\text { cohort }\end{array}$ & $\begin{array}{l}\text { Community } \\
\text { hospital }\end{array}$ & Children treated for appendicitis & $\begin{array}{l}\text { Utilization of preoperative CT scan, sensitivity of } \\
\text { CT scan, false-negative CT scans, delay in surgical } \\
\text { management }\end{array}$ & Moderate \\
\hline $\begin{array}{l}\text { Saito } \\
(2013)^{41}\end{array}$ & $\begin{array}{l}\text { Use and Accuracy of Diagnostic } \\
\text { Imaging by Hospital Type- }\end{array}$ & $\begin{array}{l}\text { Retrospective } \\
\text { cohort }\end{array}$ & $\begin{array}{l}\text { Community } \\
\text { hospital }\end{array}$ & $\begin{array}{l}\text { Children requiring appendectomy } \\
\text { for a preoperative diagnosis of } \\
\text { appendicitis }\end{array}$ & $\begin{array}{l}\text { Utilization of preoperative imaging, } \mathrm{CT} \text { and } \\
\text { ultrasound imaging accuracy }\end{array}$ & Moderate \\
\hline $\begin{array}{l}\text { York } \\
(2005)^{42}\end{array}$ & $\begin{array}{l}\text { The Influence of Advanced } \\
\text { Radiographic Imaging- }\end{array}$ & $\begin{array}{l}\text { Retrospective } \\
\text { cohort }\end{array}$ & $\begin{array}{l}\text { University- } \\
\text { affiliated } \\
\text { community } \\
\text { hospital }\end{array}$ & $\begin{array}{l}\text { Children with a preoperative } \\
\text { diagnosis of appendicitis }\end{array}$ & $\begin{array}{l}\text { Definitive treatment delay, perforation rates, } \\
\text { negative appendectomy rates, hospital charges, } \\
\text { time on antibiotics, postoperative complication } \\
\text { rates }\end{array}$ & Moderate \\
\hline \multicolumn{7}{|c|}{ Interventional Studies } \\
\hline \multicolumn{7}{|c|}{ Neonatal Medicine } \\
\hline $\begin{array}{l}\text { Altman } \\
(2011)^{43}\end{array}$ & $\begin{array}{l}\text { Parent Education by Maternity } \\
\text { Nurses- }\end{array}$ & Pre-post cohort & $\begin{array}{l}\text { Community } \\
\text { hospital }\end{array}$ & $\begin{array}{l}\text { Infants born at community hospitals } \\
\text { with maternity services }\end{array}$ & Abusive head trauma injuries per year & Moderate \\
\hline $\begin{array}{l}\text { Clemens } \\
(2002)^{44}\end{array}$ & $\begin{array}{l}\text { The Development of a Group B } \\
\text { Streptococcus Prevention Policy- }\end{array}$ & $\begin{array}{l}\text { Retrospective } \\
\text { cohort }\end{array}$ & $\begin{array}{l}\text { Nonacademically } \\
\text { affiliated } \\
\text { community } \\
\text { hospital }\end{array}$ & $\begin{array}{l}\text { GBS-positive or unknown mothers } \\
\text { with full-term infants }\end{array}$ & $\begin{array}{l}\% \text { GBS-positive or unknown mothers receiving } \\
\text { antepartum antibiotics, incidence of early-onset } \\
\text { GBS disease }\end{array}$ & Moderate \\
\hline $\begin{array}{l}\text { Hall } \\
(2010)^{45}\end{array}$ & $\begin{array}{l}\text { Neonatal Regionalization Through } \\
\text { Telemedicine- }\end{array}$ & Pre-post cohort & $\begin{array}{l}\text { Community } \\
\text { hospital }\end{array}$ & $\begin{array}{l}\text { All low birth weight infants born in } \\
\text { Arkansas hospitals }\end{array}$ & $\begin{array}{l}\text { Proportion of VLBW and ELBW infants delivered in } \\
\text { academic centers vs community hospitals }\end{array}$ & Low \\
\hline $\begin{array}{l}\text { Hulsey } \\
(1991)^{46}\end{array}$ & $\begin{array}{l}\text { Regionalized Perinatal Transport } \\
\text { Systems- }\end{array}$ & $\begin{array}{l}\text { Retrospective } \\
\text { cohort }\end{array}$ & $\begin{array}{l}\text { Community } \\
\text { hospital }\end{array}$ & Very low birth weight infants & $\begin{array}{l}\text { \% VLBW infants transported from community } \\
\text { hospitals to the academic center, survivorship by } \\
\text { transport type, \% transports requested within the } \\
\text { first hour of life }\end{array}$ & Moderate \\
\hline $\begin{array}{l}\text { Sable } \\
(2002)^{47}\end{array}$ & $\begin{array}{l}\text { Impact of Telemedicine on the } \\
\text { Practice of Pediatric Cardiology- }\end{array}$ & Prospective cohort & $\begin{array}{l}\text { Community } \\
\text { hospital }\end{array}$ & $\begin{array}{l}\text { Neonates requiring cardiac } \\
\text { echocardiogram }\end{array}$ & $\begin{array}{l}\text { Time to diagnostic services, number of tele- } \\
\text { echocardiogram transmissions that altered } \\
\text { patient care, number of transfers prevented, total } \\
\text { echocardiogram utilization }\end{array}$ & High \\
\hline $\begin{array}{l}\text { Wexelblatt } \\
(2015)^{48}\end{array}$ & Universal Maternal Drug Testing- & $\begin{array}{l}\text { Retrospective } \\
\text { cohort }\end{array}$ & $\begin{array}{l}\text { Community } \\
\text { hospital }\end{array}$ & Mother-infant dyads after birth & $\begin{array}{l}\% \text { positive urine tests without screening risk } \\
\text { factors that would have been missed using risk- } \\
\text { based urine drug testing, \% of associated infants } \\
\text { requiring admission to SCN for NAS }\end{array}$ & Moderate \\
\hline
\end{tabular}

including radiation dosing and frequency of preoperative imaging modalities and accuracy. ${ }^{30,39,41}$ For instance, Marin et al. found substantial variation in radiation dose across hospital types, with children's hospitals delivering lower median radiation doses than academic and community hospitals. ${ }^{39}$ Similarly, Saito et al. demonstrated increased use of radiating modalities when eval- uation was performed at community hospitals, with four times higher odds of computed tomography (CT) and five times lower odds of ultrasound use compared to a children's hospital. ${ }^{41}$

Two single-site studies also evaluated quality and safety outcomes associated with the use of radiographic imaging for pediatric appendicitis in a community hospital. ${ }^{40,42}$ For ex- 
TABLE 2. Characteristics of Included Studies (continued)

\begin{tabular}{|c|c|c|c|c|c|c|}
\hline $\begin{array}{l}\text { First Author } \\
\text { (Year) }\end{array}$ & Abbreviated Title & Study Design & $\begin{array}{l}\text { Community } \\
\text { Hospital Term(s) }\end{array}$ & Basic Population Description & Quality Outcome Measure(s) & Risk of Bias \\
\hline \multicolumn{7}{|c|}{ Pediatric Medicine } \\
\hline $\begin{array}{l}\text { Dayal } \\
(2015)^{49}\end{array}$ & $\begin{array}{l}\text { The Effect of Implementation of } \\
\text { Standardized, Evidence-Based } \\
\text { Order Sets- }\end{array}$ & Pre-post cohort & $\begin{array}{l}\text { Community } \\
\text { hospital }\end{array}$ & $\begin{array}{l}\text { Children hospitalized with asthma, } \\
\text { bronchiolitis, or pneumonia }\end{array}$ & $\begin{array}{l}\text { Number of bronchodilators prescribed, cost per } \\
\text { patient, 30-day readmission rate }\end{array}$ & Moderate \\
\hline $\begin{array}{l}\text { Krugman } \\
(2007)^{50}\end{array}$ & $\begin{array}{l}\text { Redefining the Community Pediatric } \\
\text { Hospitalist- }\end{array}$ & Pre-post cohort & $\begin{array}{l}\text { Community } \\
\text { hospital }\end{array}$ & $\begin{array}{l}\text { Children cared for in a combined } \\
\text { pediatric ED/inpatient unit }\end{array}$ & $\begin{array}{l}\text { Patient/family satisfaction, inpatient speed of } \\
\text { admission }\end{array}$ & Moderate \\
\hline $\begin{array}{l}\text { Kuhlmann } \\
(2013)^{51}\end{array}$ & $\begin{array}{l}\text { A Quality Improvement Project to } \\
\text { Improve Compliance With The Joint } \\
\text { Commission- }\end{array}$ & $\begin{array}{l}\text { Interrupted time- } \\
\text { series }\end{array}$ & $\begin{array}{l}\text { Community } \\
\text { teaching hospital }\end{array}$ & Children hospitalized with asthma & $\begin{array}{l}\text { Compliance with Children's Asthma Care core } \\
\text { measures }\end{array}$ & Moderate \\
\hline $\begin{array}{l}\text { Labarbera } \\
(2013)^{52}\end{array}$ & $\begin{array}{l}\text { The Impact of Telemedicine } \\
\text { Intensivist Support- }\end{array}$ & $\begin{array}{l}\text { Retrospective } \\
\text { cohort }\end{array}$ & $\begin{array}{l}\text { Community } \\
\text { hospital }\end{array}$ & $\begin{array}{l}\text { Children requiring critical care } \\
\text { consultation }\end{array}$ & $\begin{array}{l}\text { Transfer rates from community hospital to tertiary } \\
\text { care, } \% \text { transferred patients diverted from PICU } \\
\text { to ward }\end{array}$ & Moderate \\
\hline $\begin{array}{l}\text { Nkoy } \\
(2015)^{53}\end{array}$ & $\begin{array}{l}\text { Improving Pediatric Asthma Care } \\
\text { and Outcomes- }\end{array}$ & Pre-post cohort & $\begin{array}{l}\text { Urban and rural } \\
\text { general community } \\
\text { hospitals }\end{array}$ & Children hospitalized with asthma & $\begin{array}{l}\text { Composite score for eight quality measures, } \\
\text { readmission rate, hospitalization cost, hospital } \\
\text { resource utilization, ICU transfers, death }\end{array}$ & Low \\
\hline $\begin{array}{l}\text { Walia } \\
(2016)^{54}\end{array}$ & $\begin{array}{l}\text { Physician Transition of Care: } \\
\text { Benefits of I-PASS- }\end{array}$ & Pre-post cohort & $\begin{array}{l}\text { University- } \\
\text { affiliated } \\
\text { community } \\
\text { teaching hospital }\end{array}$ & $\begin{array}{l}\text { Pediatric residents participating in } \\
\text { patient handoffs in the newborn } \\
\text { nursery and pediatric inpatient ward }\end{array}$ & $\begin{array}{l}\text { Quality of patient handoffs as determined by } \\
\text { situational awareness, tangential conversation, } \\
\text { identification of illness severity, and omissions of } \\
\text { important information }\end{array}$ & High \\
\hline $\begin{array}{l}\text { Yang } \\
(2016)^{55}\end{array}$ & $\begin{array}{l}\text { Can Telemedicine Improve } \\
\text { Adherence to Resuscitation } \\
\text { Guidelines- }\end{array}$ & $\begin{array}{l}\text { Randomized } \\
\text { controlled trial }\end{array}$ & $\begin{array}{l}\text { Community } \\
\text { hospital }\end{array}$ & $\begin{array}{l}\text { Community hospital ED and } \\
\text { inpatient providers participating in } \\
\text { mock pediatric codes }\end{array}$ & $\begin{array}{l}\text { Proportion of teams who successfully defibrillated } \\
\text { within } 180 \text { seconds of pulseless ventricular } \\
\text { tachycardia }\end{array}$ & $\begin{array}{l}\text { NOS not } \\
\text { applied } \\
\text { (Experimental) }\end{array}$ \\
\hline \multicolumn{7}{|l|}{ Surgery } \\
\hline $\begin{array}{l}\text { Kelley-Quon } \\
(2015)^{56}\end{array}$ & $\begin{array}{l}\text { Academic-community partnerships } \\
\text { improve outcomes- }\end{array}$ & Pre-post cohort & $\begin{array}{l}\text { Academic- } \\
\text { sponsored } \\
\text { community facility; } \\
\text { not-for-profit } \\
\text { community } \\
\text { hospital }\end{array}$ & Traumatically injured children & $\begin{array}{l}\% \text { injured children receiving CT scans, transfers to } \\
\text { other acute facilities, in-hospital mortality }\end{array}$ & Moderate \\
\hline
\end{tabular}

Abbreviations: BPD, bronchopulmonary dysplasia; CT, computed tomography; ED, emergency department; ELBW, extremely low birth weight; GBS, group B streptococcus; ICU, intensive care unit; IV, intravenous; IVH, intraventricular hemorrhage; NAS, neonatal abstinence syndrome; NEC, necrotizing enterocolitis; NICU, neonatal intensive care unit; NOS, Newcastle-Ottawa Scale; PICC, peripherally inserted central catheter; PICU, pediatric intensive care unit; ROP, retinopathy of prematurity; SCN, special care nursery; VLBW, very low birth weight.

ample, York et al. demonstrated that, compared with nonimaged patients, patients who underwent diagnostic imaging for appendicitis experienced a significant time delay from initial evaluation to surgery and incurred significantly higher hospital charges, whereas there were no significant differences in intraoperative findings, antibiotic requirements, and surgical complications between the groups. ${ }^{42}$

\section{Interventional Studies \\ Neonatal Medicine}

We identified six studies that evaluated interventions to improve healthcare quality for neonates in community hospitals; two involved telemedicine. ${ }^{43-48} \mathrm{Hall}$ et al. described "Telenursery," a program linking regional perinatal centers with a large academic neonatal practice through real-time teleconferencing, in addition to providing weekly educational conferences. ${ }^{45}$ After its implementation, there was an increase in the state-recommended delivery of very low birth weight infants at the regional perinatal center from $24 \%$ to $33 \%(P<.05)$; clinical outcomes were not discussed. In a similar study, Sable et al. found that a videoconferencing system for cardiologists from an academic center to guide care in a community setting provided diagnostic services more quickly (28 minutes vs 12 hours) and had high diagnostic accuracy. ${ }^{47}$ The remaining four studies evaluated heterogeneous interventions such as a maternal ed- ucation program to reduce shaking injuries to infants or implementation of evidence-based order sets to reduce early onset group B streptococcal disease in neonates..$^{43,44,46,48}$ Five of the six neonatal studies demonstrated improved quality outcomes after intervention. ${ }^{43-47}$

\section{Pediatric Medicine}

Seven studies evaluated Ql interventions for children at community hospitals, ${ }^{49-55}$ three of which described interventions to improve quality of management of respiratory diseases. ${ }^{49,51,53}$ Dayal et al. evaluated the implementation of respiratory illness order sets and an asthma pathway, demonstrating a $41 \%$ reduction in asthma hospitalization cost per patient $(P<.05)$, reduced bronchodilator use for all respiratory illnesses, and no change in readmission rates. ${ }^{49}$ Similarly, Nkoy et al. evaluated an asthma "Evidence-Based Care Practice Model" implemented at seven community hospitals and demonstrated a nonsignificant reduction in readmissions $(P=$ .12), as well as lower hospitalization costs $(P=.05) .{ }^{53}$ Using $Q$ l methods, Kuhlmann et al. demonstrated improved compliance from $43 \%$ to $97 \%$ with the Asthma Home Management Plan of Care measure. ${ }^{51}$ The remaining four studies evaluated heterogeneous interventions, including telemedicine critical care consultations, use of I-PASS, and consolidation of pediatric care onto one hospital unit. ${ }^{50,52,54,55}$ Six of the sev- 
TABLE 3. Outcomes and Interventions from Included Studies within the IOM Framework

\begin{tabular}{|c|c|c|}
\hline $\begin{array}{l}\text { IOM Aim } \\
\text { of Quality } \\
\text { Healthcare }\end{array}$ & Outcomes Reported in Included Studies & Interventions Evaluated in Included Studies \\
\hline Safe & $\begin{array}{l}\text { Mortality, survivorship, incidence of potentially preventable disease (ie, shaken baby syndrome, early- } \\
\text { onset GBS, severe ROP), surgical complications, PICC line complications, radiation dosage, accuracy of } \\
\text { CT scan or ultrasound interpretation for appendicitis, accuracy of tele-echocardiography for neonatal } \\
\text { cardiac diagnoses, use of radiating CT scans during trauma or appendicitis evaluation, missed injuries } \\
\text { after trauma evaluation, cardiovascular medication errors, rates of vancomycin therapeutic drug } \\
\text { monitoring, hospital to home transition scores, patient handoff quality }\end{array}$ & $\begin{array}{l}\text { Implementation of a maternal education program, creation/use of evidence- } \\
\text { based order sets, implementation of an evidence-based care practice model, } \\
\text { policy, or care path, use of telemedicine to partner community hospitals } \\
\text { with academic centers, implementation of a neonatal transport program, } \\
\text { use of the I-PASS mnemonic during patient handoffs }\end{array}$ \\
\hline Effective & $\begin{array}{l}\text { Adherence to national, regional, or local guidelines (ie, AAP guidelines for common inpatient pediatric } \\
\text { illnesses, resuscitation guidelines for pulseless ventricular tachycardia, asthma core measures, an } \\
\text { evidence-based care practice model for inpatient asthma care, an evidence-based policy for prevention } \\
\text { of early-onset GBS, perinatal transfer guidelines, radiation dosage guidelines), narrow spectrum } \\
\text { antibiotic use for pneumonia, use of bronchodilators for bronchiolitis, provision of breastfeeding } \\
\text { support after birth, hydration status after admission with dehydration, completion of preventative } \\
\text { health measures and screening examinations for neonates prior to hospital discharge, proportion } \\
\text { of infants requiring home oxygen after ventilation, change in diagnosis/care after tele-consult with } \\
\text { specialist at an academic center, treatment for neonatal abstinence syndrome after universal maternal } \\
\text { drug screening, ICU transfers or transfers to other acute care facilities, readmission }\end{array}$ & $\begin{array}{l}\text { Creation/use of evidence-based order sets, implementation of an evidence- } \\
\text { based care practice model, policy, or care path, tele-consultation with a } \\
\text { specialist at an academic center, implementation of universal maternal } \\
\text { drug screening }\end{array}$ \\
\hline Patient-Centered & Patient/family satisfaction & Consolidation of pediatric care onto one hospital unit \\
\hline Timely & $\begin{array}{l}\text { Delays in definitive surgical treatment, inpatient speed of admission, time to defibrillation during codes, } \\
\text { time to request for transfer after birth of very low birth weight infants, time to diagnostic services }\end{array}$ & $\begin{array}{l}\text { Consolidation of pediatric care onto one hospital unit, tele-consultation } \\
\text { with a specialist at an academic center, implementation of a neonatal } \\
\text { transport program }\end{array}$ \\
\hline Efficient & $\begin{array}{l}\text { Transports prevented after telemedicine, rates of diversion from the ICU to regular floor after } \\
\text { telemedicine, rates of completion of IV antibiotics at home using PICC lines, hospital charges, projected } \\
\text { healthcare costs, use of rebound bilirubin after phototherapy, use of diagnostic tests for community } \\
\text { acquired pneumonia, duration of therapy (ie, ventilation), days on IV antibiotics after appendectomy, } \\
\text { negative appendectomy rates, hospital resource use }\end{array}$ & $\begin{array}{l}\text { Tele-consultation with a specialist at an } \\
\text { academic center, implementation of a pediatric } \\
\text { hospitalist program }\end{array}$ \\
\hline Equitable & Appendiceal perforation rates by race & \\
\hline
\end{tabular}

en pediatric studies (86\%) demonstrated improved inpatient quality outcomes after intervention, ${ }^{49,51-55}$ but only one study was multisite. ${ }^{53}$

\section{Surgery}

We identified only one study evaluating a surgical intervention aimed at improving the quality of pediatric care. ${ }^{56}$ Kelley-Quon evaluated the impact of a community hospital partnering with an Academic Medical Pediatric Trauma Center to become a Level II Pediatric Trauma Center (PTC). After achieving Level II PTC designation, they reported that children treated at the community hospital had reduced rates of CT use, transfers, and in-hospital mortality (from $81 \%$ to $51 \%, 8.5 \%$ to $2.5 \%$, and $2 \%$ to $0.4 \%$, respectively, $P<.05$ for all) compared to those treated predesignation.

\section{Radiology}

Within the domain of radiology, our review identified no interventional studies.

\section{DISCUSSION}

In this scoping review of the quality and safety of pediatric inpatient care in community hospitals, we identified 44 studies applying heterogeneous study designs and evaluating diverse patient populations and quality outcomes. We identified only one RCT in our search; all the remaining studies applied observational designs.

We found only three clinical areas that were explored in multiple studies, with consistent directionality of results: (1) perinatal regionalization, (2) telemedicine, and (3) imaging radiation. The limited evidence identified in our review suggests that delivery of early premature infants at community hospitals, rather than at tertiary hospitals, may increase risk of neonatal morbidity and/or mortality, ${ }^{13,16,46}$ that use of telemedicine may improve the effectiveness and efficiency of intensive or specialized care in community settings, ${ }^{45,47,52,55}$ and that CT use and radiation doses may be higher in community hospitals compared with other settings. ${ }^{38,39,41}$ However, even within these clinical domains, the literature was limited in amount and heterogeneous; additional research is needed to systematically review the effect of individual interventions or particular community hospital quality outcomes compared with other hospital types.

Our search identified only 14 studies evaluating $\mathrm{Q}$ I interventions within community hospitals over the almost 30 -year review period. Although limited in number, $86 \%$ of these demonstrated improvements in healthcare quality and safety, providing a positive "proof of concept" that pediatric care in community hospitals can be improved by multidisciplinary efforts and be sustained over time..$^{43-47,49,51-56}$ As pediatric depart- 
ments within community hospitals may have limited resources, aligning pediatric $\mathrm{Ol}$ efforts with adult initiatives within the same hospitals could prove advantageous. However, we did not identify any studies meeting our inclusion criteria that took this approach. Alternatively, Ql collaboratives across structurally diverse hospitals may provide valuable infrastructure for $\mathrm{Q}$ in community hospitals. For example, the Value in Inpatient Pediatrics Network has conducted several multisite Ql initiatives that have engaged both children's and community hospitals. ${ }^{57-60}$ However, none of these studies have reported community hospital-specific quality outcomes, resulting in their exclusion from this review. In future study, researchers may consider separating community hospital results from those of pediatric hospitals to highlight the effect of community hospital-specific $\mathrm{Ql}$ efforts and to allow valuable direct comparisons between hospital types.

Many studies identified in our scoping review were conducted at single hospitals. Sample sizes were often very small and power calculations were rarely reported, raising questions about the validity of the "nonsignificant" differences reported by some. Although such single-center studies provide valuable information for local improvement of inpatient care, by design the findings from these studies are unlikely generalizable to other community hospital systems. Without inclusion of another hospital type and/or quality measures with clear national benchmarks for comparison, the additional conclusions that can be drawn from these studies are limited. In comparison, many of the multicenter studies had large study samples, but more than half used data registries with limited data to evaluate outcomes, clinical context, or important covariates. ${ }^{13,20,21,25,27-30,34,35,37,39}$ The most frequently reported outcomes in these data sets-hospital charges and lengths of stay-are of limited utility in understanding healthcare quality without clear benchmarks. As a result, the evidence-base from which to draw conclusions about quality and safety in community hospitals is very limited.

Therefore, our review highlights a great need for additional research in community hospital medicine and the need for high-quality evidence generation. Risk of bias was moderate or high for the majority of included studies because of inadequate risk adjustment or statistical analysis. In the future, multicenter collaborations may help to connect research methodologists with community hospital teams to aid in the application of robust study designs and analytic techniques. Multisite collaboration may also overcome the limitation of small sample sizes that are a reality at many community hospitals.

Our study findings must be considered in the setting of several methodological limitations. The lack of a standard definition for a community hospital has led to inconsistent terms and hospital definitions used in the literature. It is possible that while following our systematic approach to define a community hospital, we inadvertently missed relevant studies that used different terms. We also excluded unpublished articles. Given that publication bias tends to favor studies with significant associations, it is possible that some studies with insignificant changes in quality outcomes were missed. Finally, in our exclusion of all non-US studies, we may have unknowingly missed literature from countries with community hospital definitions similar to those in the United States.

\section{CONCLUSIONS}

Recognizing that more than half of all children admitted to hospitals in the US receive their care at community hospitals, understanding healthcare quality in community hospitals is important. This scoping review underscores the need for additional research and higher quality evidence to determine the quality of pediatric inpatient care in these settings and identifies some particularly wide gaps that could be targeted in future research. Acknowledging that further research is necessary to address all aims of quality healthcare, markedly few studies have examined timeliness, equity, or patient-centeredness. Collaborations between academic medical centers and community hospitals may be an effective means to connect researchers with community hospital clinical teams to facilitate the application of robust study designs and analytic approaches and to facilitate multisite investigations. Research in this field would benefit from a standardized definition of a community hospital that could be consistently applied in research and $\mathrm{Q}$ l endeavors.

Disclosures: The authors have no potential conflicts of interest to disclose.

Funding: Jana Leary was supported by the National Center for Advancing Translational Sciences, National Institutes of Health, Grant Number 5TL1TR0001062-03. JoAnna Leyenaar was supported by the Agency for Healthcare Research and Quality (K08HSO24133).

Disclaimer: The content is solely the responsibility of the authors and does not necessarily represent the official views of the $\mathrm{NIH}$ or the AHRQ.

\section{References}

1. McGlynn EA, Asch SM, Adams J, et al. The quality of health care delivered to adults in the United States. N Engl J Med. 2003;348(26):2635-2645. https:// doi.org/10.1056/NEJMsa022615

2. Richardson WC, Berwick DM, Bisgard C, et al. To err is human: building a safer health system-Institute of Medicine. Medscape. http://www.iom.edu/ Reports/1999/To-Err-is-Human-Building-A-Safer-Health-System.aspx; 2000. Accessed October 7, 2016.

3. Classen DC, Resar R, Griffin F, et al. 'Global Trigger Tool' shows that adverse events in hospitals may be ten times greater than previously measured. Health Aff. 2011:30(4):581-589. https://doi.org/10.1377/hlthaff.2011.0190.

4. Stockwell DC, Landrigan CP, Schuster MA, et al. Using a pediatric trigger tool to estimate total harm burden hospital-acquired conditions represent. Pediatr Qual Saf. 2018:3(3):e081. https://doi.org/10.1097/pq9.0000000000000081.

5. Institute of Medicine (US) Committee on Quality of Health Care in America. Crossing the Quality Chasm. Washington (DC): National Academies Press (US); 2001. https://doi.org/10.17226/10027.

6. Rauch DA, Lye PS, Carlson D, et al. Pediatric hospital medicine: a strategic planning roundtable to chart the future. J Hosp Med. 2012;7(4):329-334. https://doi.org/10.1002/jhm.950.

7. Simpson L, Fairbrother G, Hale S et al. Reauthorizing SCHIP: Opportunities for Promoting Effective Health Coverage and HighQuality Care for Children and Adolescents. (Publication 1051). Commonw Fund; 2007.

8. Percelay JM. Pediatric hospitalists working in community hospitals. Pediatr Clin North Am. 2014;61(4):681-691. https://doi.org/10.1016/j.pcl.2014.04.005.

9. Leyenaar JK, Ralston SL, Shieh MS et al. Epidemiology of pediatric hospitalizations at general hospitals and freestanding children's hospitals in the United States. J Hosp Med. 2016;11(11):743-749. https://doi.org/10.1002/jhm.2624.

10. Peters MDJ, Godfrey CM, Khalil H et al. Guidance for conducting systematic scoping reviews. Int J Evid Based Healthc. 2015;13(3):141-146. https://doi. org/10.1097/XEB.0000000000000050. 
11. Tricco AC, Lillie E, Zarin W, et al. PRISMA extension for scoping reviews (PRIS MA-ScR): checklist and explanation. Ann Intern Med. 2018;169(7):467-473. https://doi.org/10.7326/M18-0850

12. Alobaidi R, Morgan C, Basu RK, et al. Association between fluid balance and outcomes in critically ill children: a systematic review and meta-analysis. JAMA Pediatr. 2018;172(3):257-268. https://doi.org/10.1001/jamapediatrics.2017.4540

13. Cifuentes J, Bronstein J, Phibbs CS et al. Mortality in low birth weight in fants according to level of neonatal care at hospital of birth. Pediatrics. 2002;109(5):745-751. https://doi.org/10.1542/peds.109.5.745.

14. Donohue PK, Hussey-Gardner B, Sulpar LJ, Fox R, Aucott SW. Convalescent care of infants in the neonatal intensive care unit in community hospitals: risk or benefit? Pediatrics. 2009;124(1):105-111. https://doi.org/10.1542/ peds.2008-0880

15. Izatt SD. Breastfeeding counseling by health care providers. J Hum Lact. 1997;13(2):109-113. https://doi.org/10.1177/089033449701300210.

16. Kuo S, Kimata C, Akamine K, Young B, Balaraman V Outcomes of inborn and transported extremely premature very-low-birthweight infants in Hawai'i. Pediatr Int. 2012;54(3):365-369. https://doi.org/10.1111/j.1442 200X.2012.03561.x.

17. Maisels MJ, Kring E. Rebound in serum bilirubin level following intensive phototherapy. Arch Pediatr Adolesc Med. 2002;156(7):669-672. https://doi. org/10.1001/archpedi.156.7.669.

18. Meadow W, Mendez D, Makela J et al. Can and should level II nurseries care for newborns who require mechanical ventilation? Clin Perinatol. 1996:23(3):551-561. https://doi.org/10.1016/S0095-5108(18)30227-6.

19. Phibbs CS, Mortensen L. Back transporting infants from neonatal intensive care units to community hospitals for recovery care: effect on total hospita charges. Pediatrics. 1992;90(1):22-26.

20. Wall SN, Handler AS, Park CG. Hospital factors and nontransfer of small babies: A marker of deregionalized perinatal care? J Perinatol. 2004;24(6):351 359. https://doi.org/10.1038/sj.jp.7211101.

21. Alexander DC, Bundy DG, Shore AD et al. Cardiovascular medication errors in children. Pediatrics. 2009;124(1):324-332. https://doi.org/10.1542/ peds.2008-2073.

22. Balch AH, Constance JE, Thorell EA, et al. Pediatric vancomycin dosing: trends over time and the impact of therapeutic drug monitoring. J Clin Pharmacol. 2015;55(2):212-220. https://doi.org/10.1002/jcph.402

23. Conway PH, Edwards S, Stucky ER et al. Variations in management of com mon inpatient pediatric illnesses: hospitalists and community pediatricians. Pediatrics. 2006;118(2):441-447. https://doi.org/10.1542/peds.2006-0484.

24. Frank BS, Pollack MM. Quantitative quality assurance in a community hospital pediatric intensive care unit. West J Med. 1992;157(2):149-151.

25. Leyenaar JK, Lagu T, Shieh MS, Pekow PS, Lindenauer PK. Variation in resource utilization for the management of uncomplicated community-acquired pneumonia across community and children's hospitals. J Pediatr. 2014;165(3):585-591. https://doi.org/10.1016/j.jpeds.2014.04.062.

26. Leyenaar JK, Desai AD, Burkhart $Q$, et al. Quality measures to assess care transitions for hospitalized children. Pediatrics. 2016;138(2):e20160906. https://doi.org/10.1542/peds.2016-0906.

27. Meurer JR, Kuhn EM, George V, Yauck JS, Layde PM. Charges for childhood asthma by hospital characteristics. Pediatrics. 1998;102(6):E70. https://doi. org/10.1542/peds.102.6.e70.

28. Myers J, Lehna C. Where are lengths of stay longer and total charges higher for pediatric burn patients? J Burn Care Res. 2014;35(5):382-387. https://doi. org/10.1097/BCR.0000000000000012.

29. Myers J, Smith M, Woods C, Espinosa C, Lehna C. The effect of transfers between health care facilities on costs and length of stay for pediatric burn patients. J Burn Care Res. 2015;36(1):178-183. https://doi.org/10.1097/ BCR.0000000000000206

30. Odetola FO, Gebremariam A, Freed GL. Patient and hospital correlates of clinical outcomes and resource utilization in severe pediatric sepsis. Pediatrics. 2007;119(3):487-494. https://doi.org/10.1542/peds.2006-2353.

31. Scherb CA Stevens MS, Busman C. Outcomes related to dehydration in the pediatric population. J Pediatr Nurs. 2007:22(5):376-382. https://doi. org/10.1016/j.pedn.2006.10.004

32. Van Winkle $\mathrm{P}$, Whiffen T, Liu IL. Experience using peripherally inserted centra venous catheters for outpatient parenteral antibiotic therapy in children at a community hospital. Pediatr Infect Dis J. 2008;27(12):1069-1072. https://doi. org/10.1097/INF.0b013e31817d32f2.

33. Beaty JS, Chendrasekhar A, Hopkins J, Gruelke L. Missed injuries in pediatric trauma patients. J App/ Res. 2003;3(1):84-88. https://jrnlappliedresearch com/articles/Vol3lss1/CHENDRASEKHAR.htm. Accessed July 8, 2019.

34. Kelley-Quon LI, Tseng CH, Jen HC, Shew SB. Hospital type predicts surgical complications for infants with hypertrophic pyloric stenosis. Am Surg 2012;78(10):1079-1082.

35. Kelley-Quon LI, Tseng CH, Jen HC, Lee SL, Shew SB. Hospital type as a metric for racial disparities in pediatric appendicitis. J Am Coll Surg. 2013;216(1):74 82. https://doi.org/10.1016/j.jamcollsurg.2012.09.018.

36. Pokala N, Sadhasivam S, Kiran RP, Parithivel V. Complicated appendicitis-is the laparoscopic approach appropriate? A comparative study with the open approach: outcome in a community hospital setting. Am Surg. 2007;73(8):732-737.

37. Smith JT, Price C, Stevens PM, Masters KS, Young M. Does pediatric orthopedic subspecialization affect hospital utilization and charges? J Pediatr Orthop. 1999;19(4):553-555. https://doi.org/10.1097/01241398-19990700000027

38. Calvert C, Strauss KJ, Mooney DP. Variation in computed tomography radiation dose in community hospitals. J Pediatr Surg. 2012;47(6):1167-1169. https://doi.org/10.1016/j.jpedsurg.2012.03.021

39. Marin JR, Sengupta D, Bhargavan-Chatfield M et al. Variation in pediatric cervical spine computed tomography radiation dose index. Acad Emerg Med. 2015;22(12):1499-1505. https://doi.org/10.1111/acem.12822.

40. Reich JD, Brogdon B, Ray WE, Eckert J, Gorell H. Use of CT scan in the diagnosis of pediatric acute appendicitis. Pediatr Emerg Care. 2000;16(4):241243. https://doi.org/10.1097/00006565-200008000-00006

41. Saito JM, Yan Y, Evashwick TW, Warner BW, Tarr PI. Use and accuracy of diagnostic imaging by hospital type in pediatric appendicitis. Pediatrics. 2013;131(1):e37-e44. https://doi.org/10.1542/peds.2012-1665.

42. York D, Smith A, Phillips JD, Von Allmen D. The influence of advanced radiographic imaging on the treatment of pediatric appendicitis. J Pediatr Surg. 2005;40(12):1908-1911. https://doi.org/10.1016/j.jpedsurg.2005.08.004.

43. Altman RL, Canter J, Patrick PA et al. Parent education by maternity nurses and prevention of abusive head trauma. Pediatrics. 2011;128(5):e1164-e1172. https://doi.org/10.1542/peds.2010-3260.

44. Clemens CJ, Gable EK. The development of a group B streptococcus prevention policy at a community hospital. J Perinatol. 2002;22(7):523-525. https://doi.org/10.1038/sj.jp.7210794.

45. Hall RW, Hall-Barrow J, Garcia-Rill E. Neonatal regionalization through telemedicine using a community-based research and education core facility. Ethn Dis. 2010;20(1 Suppl 1):S136-S140.

46. Hulsey TC, Pittard WB 3rd, Ebeling M. Regionalized perinatal transport systems: association with changes in location of birth, neonatal transport, and survival of very low birth weight deliveries. J S C Med Assoc. 1991;87(12):581-584.

47. Sable CA, Cummings SD, Pearson GD, et al. Impact of telemedicine on the practice of pediatric cardiology in community hospitals. Pediatrics. 2002;109(1):E3. https://doi.org/10.1542/peds.109.1.e3.

48. Wexelblatt SL, Ward LP, Torok $\mathrm{K}$ et al. Universal maternal drug testing in a high-prevalence region of prescription opiate abuse. J Pediatr. 2015;166(3):582-586. https://doi.org/10.1016/j.jpeds.2014.10.004.

49. Dayal A, Alvarez F. The effect of implementation of standardized, evidence-based order sets on efficiency and quality measures for pediatric respiratory illnesses in a community hospital. Hosp Pediatr. 2015;5(12):624-629. https://doi.org/10.1542/hpeds.2015-0140.

50. Krugman SD, Suggs A, Photowala HY, Beck A. Redefining the community pediatric hospitalist: the combined Pediatric ED/Inpatient Unit. Pediatr Emerg Care. 2007:23(1):33-37. https://doi.org/10.1097/01 pec.0000248685.94647.01.

51. Kuhlmann S, Mason B, Ahlers-Schmidt CR. A quality improvement project to improve compliance with the joint commission children's asthma care-3 measure. Hosp Pediatr. 2013;3(1):45-51. https://doi.org/10.1542/ hpeds.2012-0015

52. Labarbera JM, Ellenby MS, Bouressa P et al. The impact of telemedicine intensivist support and a pediatric hospitalist program on a community hospital. Telemed J E Health. 2013;19(10):760-766. https://doi.org/10.1089/ tmj.2012.0303

53. Nkoy F, Fassl B, Stone B, et al. Improving pediatric asthma care and outcomes Across multiple hospitals. Pediatrics. 2015;136(6):e1602-e1610. https://doi.org/10.1542/peds.2015-0285

54. Walia J, Qayumi Z, Khawar N, et al. Physician transition of care: benefits of I-PASS and an electronic handoff system in a community pediatric residency program. Acad Pediatr. 2016;16(6):519-523. https://doi.org/10.1016/j. acap.2016.04.001.

55. Yang $C P$, Hunt EA, Shilkofski $N$ et al. Can telemedicine improve adherence to resuscitation guidelines for critically ill children at community hospitals? A randomized controlled trial using high-fidelity simulation. Pediatr Emerg Care. 2017;33(7):474-479. https://doi.org/10.1097/PEC.0000000000000653.

56. Kelley-Quon LI, Crowley MA, Applebaum H, et al. Academic-community partnerships improve outcomes in pediatric trauma care. J Pediatr Surg. 2015;50(6):1032-1036. https://doi.org/10.1016/j.jpedsurg.2015.03.033.

57. Ralston S, Garber M, Narang S, et al. Decreasing unnecessary utilization in acute bronchiolitis care: results from the value in inpatient pediatrics network. J Hosp Med. 2013;8(1):25-30. https://doi.org/10.1002/jhm.1982.

58. Ralston SL, Garber MD, Rice-Conboy E, et al. A multicenter collaborative to reduce unnecessary care in inpatient bronchiolitis. Pediatrics. 2016;137(1):e20150851. https://doi.org/10.1542/peds.2015-0851.

59. Parikh K, Biondi E, Nazif J, et al. A multicenter collaborative to improve care of community acquired pneumonia in hospitalized children. Pediatrics. 2017;139(3):e20161411. https://doi.org/10.1542/peds.2016-1411.

60. Leyenaar JK, Bergert L, Mallory LA, et al. Pediatric primary care providers' perspectives regarding hospital discharge communication: a mixed methods analysis. Acad Pediatr. 2015;15(1):61-68. https://doi.org/10.1016/j. acap.2014.07.004 